\title{
Study on new gray transformation of infrared image based on visual property
}

\author{
Shaosheng DAI ${ }^{1}$, Xingfu $\mathrm{LI}^{2}$, Zhihui $\mathrm{DU}^{3}$, Bin ZhANG ${ }^{4}$ and Xinlin $\mathrm{HUANG}^{5}$
}

\begin{abstract}
Since the infrared image has disadvantages such as narrow gray distribution, low contrast and blurred vision effects, a new method of gray transformation of infrared image based on human visual property is proposed. The method aims to transform the gray distribution of infrared object to the sensitive regions of human visual system to enhance display effects of infrared images, which takes advantage of the feature that human visual system has different ability of visual resolution for infrared object information under different gray background. Experiment results show that the processes infrared images have rich details, clear outline and more suitable for observation.
\end{abstract}

Keywords: Human visual property; Infrared image; Gray transformation

\section{Introduction}

Infrared image is different from visible image which has low background and high contrast, since useful signal generally is mixed in the background resulting in low contrast, poor visual effects and limiting application of infrared imaging technology[1]. Therefore, infrared images usually need enhancement in the practical project to meet the requirements of further processing such as infrared target detection, tracking and identification[2].

Traditional methods to enhance infrared images include linear gray stretch and histogram equalization enhancement. Linear gray stretch method merely transforms gray values of the infrared image to the range $[0,255]$ according to the linear mapping relationships to improve the contrast of the image[3]. Histogram equalization method changes the contrast by considering the entire image information. It can make the image obtain a higher contrast by compressing grayscale with the fewer number of pixels and extending one with more number of pixels[4]. Both of these two methods process the image itself rather than consider the requirement

\footnotetext{
${ }^{3}$ Zhihui DU( $\left.\square\right)$

Chongqing Key Laboratory of Signal and Information Processing, Chongqing University of

Posts and Telecommunications, Chongqing, China

e-mail: eliteduzhihui@outlook.com
} 
of human visual characteristics. Since the final effects of infrared image processing is observed by the human eyes which have special visual requirements of brightness of the background and objectives of the infrared images. For instance, human eyes gain different details of the same target due to the brightness of the background. Meanwhile, human evaluate the image quality.

Therefore, this paper provides a method of gray transforms which is based on human visual characteristics to enhance the visual effects.

\section{Human Visual Characteristics}

Visual research shows that the response of the human eyes to the changes of the light brightness is nonlinear[5]. Usually the minimum light intensity to enable human eyes just to distinguish the light difference is called the visibility threshold of the brightness. In other words, visual system fails to perceive the change of light brightness in the certain range when light brightness increases. It can sense the change only when the light brightness increases $\mathrm{I}+\Delta \mathrm{I}$ which is called contrast sensitivity[6].

We use the image as the Figure 1 shows to study the threshold of changes of the brightness in different light brightness background and establish the model of the human visual change with the light intensity. In Figure 1, the gray scale of the background and the object can be adjusted and can change in the range of $[0,255]$. First of all, set the gray scale of the background to 0 , increase the gray scale by 1 from 0 until the object can be observed clearly and record the gray scale of the object in the background with 0 grey scale. Repeat the process until the gray scale of background is set to 255. Depict the curve as shown in Figure 2 according to the records. Figure 2 is visual resolving ability curve.

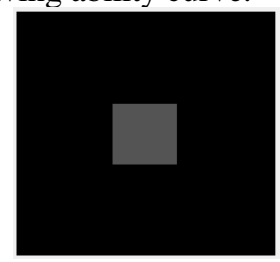

Fig. 1 Object in the different background

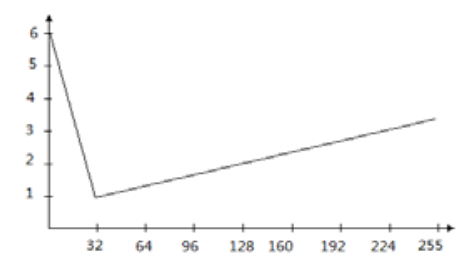

Fig. 2 Visual resolving ability curve 
Figure 2 indicates that human eyes have a poor resolving ability of gray scale when the gray scale of the background is big or small and they have a satisfying ability when the gray scale of the background is in the middle range. For example, human eyes can hardly distinguish the object in the background with gray scale being 0 and can observe the object when the gray scale increases up to 6. Meanwhile, human eyes have the strongest resolving ability near 32 and they only can observe the object when the gray scale of object is 3 near 255 .

We can build the piecewise function according to Figure 2:

$$
\Delta y=\left\{\begin{array}{lc}
-\frac{5}{32} x+6 & 0 \leq x<32 \\
\frac{2}{223} x+\frac{159}{223} & 32 \leq x<255
\end{array}\right.
$$

In this equation, $\Delta y$ is visual resolving ability and $x$ is grey scale.

\section{Gray scale transformation based on visual resolving ability}

We can process the infrared image according to the visual characteristic as the following steps. First of all, we move the gray scale range of infrared image to the region of the gray scale being 32. Then we transform the gray scale of infrared image based on visual resolving ability curve and make it cover from [0, 255].

In order to process the infrared image, we need to build the model of infrared image gray scale transformation. Function 1 shows the relationship between visual resolving ability and gray scale. We can obtain Function 2 by get reciprocal of Function 1 as follows:

$$
y_{d}=\left\{\begin{array}{lr}
\frac{32}{192-5 x} & 0 \leq x<32 \\
\frac{223}{2 x+159} & 32 \leq x<255
\end{array}\right.
$$

Where $y_{d}$ is the reciprocal of $\Delta y$, that is the gray scale which make human eyes observe the object in the background with the gray scale $x$. Then integrate Function 2 and get Function 3 which is the gray scale of the whole infrared image observed clearly:

$$
y_{w}=\left\{\begin{array}{cr}
-\frac{32}{5} \ln (192-5 x) & 0 \leq x<32 \\
\frac{223}{2} \ln (2 x+159) & 32 \leq x<255
\end{array}\right.
$$


Where $y_{w}$ is the resolving gray scale after processing, if the gray scale range of the input infrared image is $\left[x_{\min }, x_{\max }\right]$, the resolving gray $\mathrm{G}$ of the whole image can be written as follows:

$$
G=\sum_{x_{\min }}^{x_{\max }} y_{w}
$$

Assume that the gray scale range expands to $\left[x_{\min }^{\prime}, x_{\max }^{\prime}\right]$ after processing. Then the multiple of expanding is:

$$
K=\frac{x_{\max }^{\prime}-x_{\min }^{\prime}}{G}
$$

Function 5 can be simplified as functio6 when the gray scale range is $[0,255]$.

$$
K=\frac{255}{G}
$$

We can gain the infrared image gray scale transformation function combining function 3, 4 and 6 .

$$
y_{t}=\left\{\begin{array}{lr}
K\left[-\frac{32}{5} \ln (192-5 x)\right] & 0 \leq x<32 \\
K\left[\sum_{0}^{32} y_{t}+\frac{223}{2} \ln (2 x+159)\right] & 32 \leq x<255
\end{array}\right.
$$

Where $y_{t}$ refer to gray scale of infrared image after transformation, Function 7 considers the visual resolving ability into the gray scale transformation. According to the logarithmic relationship between the gray scale transformation $y_{t}$ and input gray scale $x$, we can conclude that logarithmic transformation expands the original gray scale information in the gray scale range of human eyes having satisfying resolving ability; it compresses the original gray scale information in the gray scale range of eyes having poor resolving ability. In this way, we can establish gray scale transformation curve for observation easily.

\section{Experimental Results}

We took an infrared image and chose it to conduct experiment to verify the algorithm. The experiment results are shown in Figure 3. Figure 3(a) is the original infrared image and the histogram. Figure $3(\mathrm{~b})$ is the image after gray scale transformation based on histogram equalization enhancement and its histogram. Figure $3(c)$ is the image after gray scale transformation based on visual property and its histogram. 

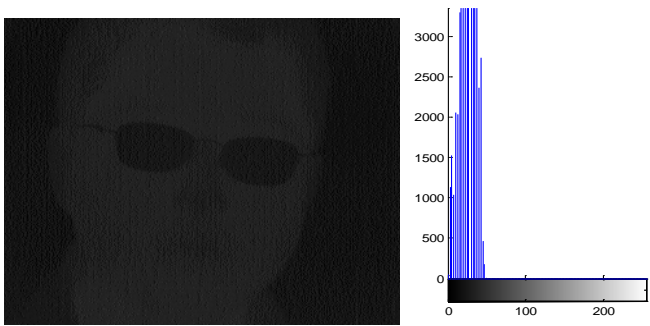

(a)Original infrared image and its histogram
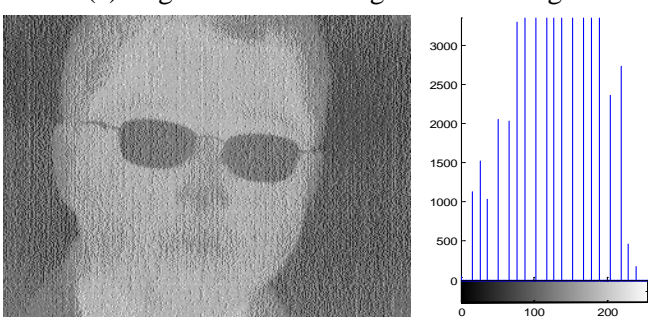

(b)Image after gray scale transformation based on histogram equalization enhancement and its histogram
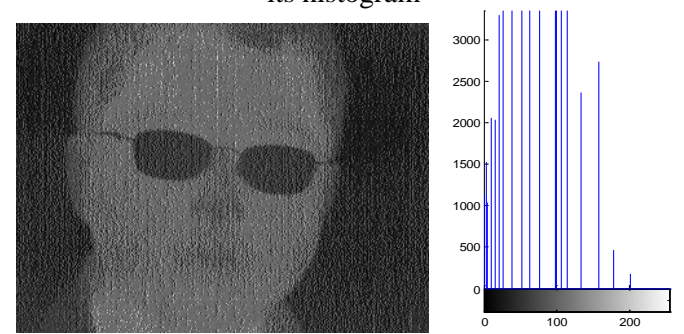

(c)Image after gray scale transformation based on visual property and its histogram

Fig. 3 Effect comparison of gray scale transformation based on histogram equalization enhancement and based on visual property.

Figure 3(a) has a narrow range of gray scale distribution which almost all the pixels distribute between $[0,47]$. We can hardly observe the face in the background under the poor contrast. The gray scale almost cover $[0,255]$ in the Figure 3 (b) with the traditional method and distributes uniformity; however, the image is lighter and the outline of the object is not clear. We can't obtain more details. While the gray scale distributes between[0, 200] in the Figure 3(c) with the new method. The image has better effects with rich details and more information. Comparing Figure 3(b) and Figure 3(c), we draw a conclusion that gray scale distribution of infrared images based on visual property mainly distributes in visual sensitive region and gray scale distributes uniformly in $[20,120]$ which can show the outline of the face clearly with medium contrast. 


\section{Conclusions}

The paper firstly studies human eyes resolving ability and then builds the logarithmic model of infrared image gray scale transformation. The model can transform the input original image to the region human eyes are sensitive to with the help of visual property. The experiment results indicate that infrared image gray scale transformation based on visual property can gain more information and better visual effects. Meanwhile, it can be easily realized. It will have bright future in the practical projects.

\section{Acknowledgements}

This work is supported by the National Natural Science Foundation of China (No.61275099, No.61102131), the Project of Key Laboratory of Signal and Information Processing of Chongqing (No.CSTC2009CA2003), the Natural Science Foundation of Chongqing Science and Technology Commission (No.CSTC2010BB2411, CSTC2010BB2398, CSTC2006BB2373), the Natural Science Foundation of Chongqing Municipal Education Commission (No.KJ060509, KJ080517), the Science and technique foundation of Chongqing (CSTC, 2011AB2008), and the Natural Science Foundation of Chongqing University of Posts and Telecommunications (CQUPT) (A2006-04, A2006-86).

\section{References}

1. JI TL, SUND AREHAN MK and Roehrig H (1994). Adaptive image contrast enhancement based on human visual properties. Medical Imaging, IEEE Trans Medical Imaging, 13(4), 573-586.

2. ZHANG Zhi-zhong, KANG Rong, ZHENG Wei-ping, HAN Yi and REN Li-na (2009). An Infrared Image Enhancement Method Based on a Combination of Methods. Infrared Technology, 31(10), 591-593.

3. ZHANG Xiao, BAI Ting-zhu, LUO Xiao and HE Yu-qing (2008). IR Image Mapping Based on Human Visual Gray-scale Properties. Infrared Technology, 30(4), 225-229.

4. Jung CR, Scharcanski J (2003). Adaptive image denoising and edge enhancement in scalespace using the wavelet transform. Pattern Recognition Letters, 24(7), 965-971.

5. DING Xu-xing, ZHU Ri-hong and LI Jian-xin (2004). A Criterion of Image Quality Assessment Based on Property of HVS. Journal of Image and Grophics, 9(2), 191-194.

6. WANG Xiang-hui, ZENG Ming (2008). A new metric for objectively assessing the quality of enhanced images based on human visual perception. Journal of Optoelectronics • Laser, 19(2), 258-262. 